\title{
ON COEFFICIENT FIELDS
}

\author{
by A. GEDDES
}

(Received 12th September, 1958)

1. Introduction. Let $Q$ be a complete local ring which has the same characteristic as its residue field $P$, and, for the present, let us denote by $\bar{A}$ the image of a subset $A$ of $Q$ under the natural homomorphism of $Q$ onto $P$. Then a subfield $F$ of $Q$ is called a coefficient field if $\bar{F}=P$. It has been shown in [2] and in [3] that a complete equicharacteristic local ring, such as the above, always possesses at least one coefficient field; this is the embedding theorem for the equicharacteristic case.

In the present paper, we shall be concerned with the following more general question. When can a given subfield $K$ of $Q$ be extended to a coefficient field? Cohen [2] has constructed examples to show that it is not always possible to extend $K$ in this way.

To give full force to our results, we shall deal with a situation which is more general than the above in two respects.

Firstly, we shall establish our results for complete weak local rings rather than for complete local rings. A complete weak local ring, we recall, is a ring $Q$ (commutative and with an identity element) in which the non-units form an ideal $\mathfrak{m}$ such that $\bigcap_{i=1}^{\infty} m^{i}=(0)$, and which is complete with respect to the topology imposed on it by taking the powers of $m$ as a fundamental system of neighbourhoods of zero.

Secondly, we shall suppose that, besides being given a subfield $\dagger K$ of the complete weak local ring $Q$, we are also given a subfield $F_{1}$ of $P$ which contains $\bar{K}$, and shall consider conditions under which a subfield $F$ of $Q$ can be found, which is an extension field of $K$ and such that $\bar{F}=F_{1}$.

It will be noted that, if we take $Q$ to be a complete local ring with $F_{1}=P$, then our considerations reduce to those described above, concerning the extension of $K$ to a coefficient field of $Q$. We shall see later that, by further specializing our data, we can arrange for the embedding theorem to emerge as a particular case of our main result.

In what follows, we shall make use of the notion of separability. We recall that an extension field $F$ of a field $K$ is separable over $K$, in the classical sense, if $F$ is an algebraic extension of $K$, and if the minimal polynomial over $K$ of any element of $F$ has distinct roots. It may be shown (see, e.g., [5], p. 113) that a generalization of this concept is obtained if we make the

Definimion. An extension field $F$ of a field $K$ is separable over $K$ if either

(i) $K$ has characteristic zero, or

(ii) $K$ has prime characteristic $p$, and the following condition is satisfied : given any elements $x_{1}, x_{2}, \ldots, x_{m}$ of $F$ which are linearly independent over $K$, their $p$-th powers $x_{1}^{p}, x_{2}^{p}, \ldots, x_{m}^{p}$ are also linearly independent over $K$.

This concept of separability is much more general than the classical one; e.g., it can be

† If $Q$ is a weak local ring and if $S$ is a subring which has an identity element (in particular, if $S$ is a subfield of $Q$ ), then the identity of $S$ coincides with that of $Q$, since (see [4], foot-note) $Q$ has no non-trivial idempotent elements. 
shown (see, e.g., [5], p. 113) that, according to this definition, all pure transcendental extensions of a given field $K$ are separable extensions of $K$. From now on, we shall use the term " separable " in the sense just described.

In this paper we shall prove that, if (as above) $F_{1}$ is separable over $\bar{K}$, then $K$ can be extended to a field $F$ with the required properties ; and, in particular, that $K$ can be extended to a coefficient field when $P$ is separable over $\bar{K}$. It must be pointed out, however, that, while the condition of separability takes account of a large number of cases, it is not a necessary condition for an extension of the desired type to be possible. This may be seen by taking $F$ to be an inseparable extension of a field $K$, and considering $F[[X]]$, the ring of formal power series in an indeterminate $X$ over $F$; this complete local ring has $F$ as a coefficient field containing $K$, and the separability condition is not satisfied.

2. Weak primary rings. A weak primary ring of exponent $\rho([3],[4])$ is a complete weak local ring, with maximal ideal $p$ (say), in which $p^{\rho}=(0), p^{\nu} \neq(0)$ if $\nu<p$.

For any such ring $R$ of prime characteristic $p$, we shall denote by $R^{y}$ the subring of $R$ consisting of all elements which have a $p$-th root in $R$. Further, if $K$ is any subfield of $R$, we shall write $K R^{p}$ for the smallest subring of $R$ which contains $K$ and $R^{p}$; this subring clearly consists of all elements of $R$ expressible in the form

$$
a_{1} x_{1}^{p}+a_{2} x_{2}^{p}+\ldots+a_{n} x_{n}^{p} \quad\left(a_{i} \in K, x_{i} \in R\right) .
$$

LEMMA 1. Let $R$, a weak primary ring with maximal ideal $p$, have prime characteristic $p$ and exponent $\rho$, where $\mathrm{I}<\rho \leqslant p$. Let $K$ be a subfield of $R$. Then $K$ can be extended to a cofficient field of $R$ if and only if

$$
K R^{p} \cap \mathcal{P}=(0) .
$$

Proof. It has been shown [3] that, under the stated conditionst, $R^{p}$ is a subfield of $R$ and that the coefficient fields of $R$ are precisely the maximal subfields of $R$ which contain $R^{p}$.

If, then, $K$ can be extended to a coefficient field $F$, we have $K R^{p} \subseteq F$, so that

$$
K R^{p} \cap \mathfrak{p} \subseteq F \cap \mathfrak{p}=(0) .
$$

This establishes the necessity of the condition.

For the sufficiency, we note that the condition (1) implies that $K R^{p}$ is an integral domain ; and $R$ contains the quotient field of $K R^{p}$. A simple application of Zorn's Lemma to the set of all subfields of $R$ which contain this quotient field (partially ordered by inclusion) now establishes the existence of a maximal subfield of $R$ containing the quotient field. This maximal subfield contains $K$ and $R^{p}$, and hence is a coefficient field of the required type.

In the lemma which follows, we use the bar notation for residues modulo $p$.

LEMma 2. Let $K$ be a subfield of a weak primary ring $R$ of exponent $\rho$, where $\rho \leqslant 2$ in the case of characteristic zero and $\rho \leqslant p$ in the case of characteristic $p(>0)$. Assume that the residue field $E$ of $R$ is a separable extension of $\bar{K}$. Then $K$ can be extended to a coefficient field of $R$.

Proof. We first observe that, if $\rho=1$, then $R$ is a field, and the result is trivial.

$\dagger \operatorname{In}[3]$, only $\rho=2$ was considered. The proof extends at once, however, to the case in which $1<p \leqslant p$. In the next section, we shall require this result and those of Lemmas 2, 3 and 4 only in the case of exponent 2, but it is of interest to consider here the more general situation to see to what extent these results are dependent on the exponent being " small". 


\section{A. GEDDES}

Suppose now that $R$ has characteristic zero and that $\rho=2$. Then, as was shown in [3], any maximal subfield of $R$ is a coefficient field. To complete the proof in this case, we apply Zorn's Lemma to the set of all subfields of $R$ which contain $K$, to show that there exists a maximal one.

We may now assume that $R$ has prime characteristic $p$ and that $1<\rho \leqslant p$. By Lemma 1, the result will follow if we can prove that $K R^{p} \cap p=(0), p$ being the maximal ideal of $R$.

Assume, then, that $x$ is any element of $K R^{p} \cap p$, so that, for suitable $a_{i} \in K$ and $x_{i} \in R$, we have

$$
x=\sum_{i=1}^{n} a_{i} x_{i}^{p}
$$

From the set $\left\{\bar{x}_{1}, \bar{x}_{2}, \ldots, \bar{x}_{n}\right\}$ of elements of $E$, we choose a subset consisting of elements which are linearly independent over $\bar{K}$ and such that the subset is maximal with respect to this property. We may suppose that the $\bar{x}_{i}$ are numbered in such a way that the elements so chosen are $\bar{x}_{1}, \bar{x}_{2}, \ldots, \bar{x}_{m} \quad(m \leqslant n)$. Because of the maximality of this subset, we may write

$$
\bar{x}_{i}=\sum_{j=1}^{m} \bar{b}_{i j} \bar{x}_{j} \quad\left(b_{i j} \in K ; i=1,2, \ldots, n\right) .
$$

Accordingly

$$
\left(x_{i}-\sum_{j=1}^{m} b_{i j} x_{j}\right) \in \mathfrak{p}
$$

so that, since $\rho \leqslant p$,

$$
\left(x_{i}-\sum_{j=1}^{m} b_{i j} x_{j}\right)^{p} \in \mathfrak{p}^{p} \subseteq \mathfrak{p}^{\rho}=(0) \quad(i=1,2, \ldots, n) .
$$

Thus, using the fact that $R$ has characteristic $p$, we obtain

$$
x_{i}^{p}=\sum_{j=1}^{m} b_{i j}^{p} x_{j}^{p} \quad(i=1,2, \ldots, n),
$$

and the relation (2) becomes

$$
x=\sum_{j=1}^{m} c_{j} x_{j}^{p}
$$

where

$$
c_{j}=\sum_{i=1}^{n} a_{i} b_{i j}^{p} \in K \quad(j=1,2, \ldots, m)
$$

Reading relation (3) modulo $\mathfrak{p}$ and noting that $x \in \mathfrak{p}$, we obtain in $E$

$$
0=\sum_{j=1}^{m} \vec{c}_{j} \bar{x}_{j}^{p}
$$

Now the elements $\bar{x}_{1}, \bar{x}_{2}, \ldots, \bar{x}_{m}$ are linearly independent over $\bar{K}$; consequently, by the separability of $E$ over $\bar{K}$, the elements $\bar{x}_{1}^{p}, \bar{x}_{2}^{p}, \ldots, \bar{x}_{m}^{p}$ are also linearly independent over $\bar{K}$. But $\bar{c}_{j} \in \bar{K}$, by (4), and hence (5) gives $\bar{c}_{j}=0$, so that 


$$
c_{j} \in K \cap \mathfrak{p}=(0) \quad(j=1,2, \ldots, m) \text {; }
$$

accordingly, by (3), $x=0$. This proves that the only element of $K R^{p} \cap p$ is the zero element, which is what we had to show.

If $a$ is any proper ideal of a weak primary ring (i.e. if $a$ is an ideal other than the whole ring), then $a$ is contained in the maximal ideal, and hence there exist positive integers $\mu$ such that $\mathfrak{a}^{\mu}=(0)$. We shall call the smallest such positive integer $\mu$ the exponent of $\mathfrak{a}$.

Lemma 3. Suppose that $S$ and $S^{\prime}$ are weak primary rings, and let $\sigma$ be a homomorphism of $S$ onto $S^{\prime}$ with kernel of exponent $\mu$. Then, if $F^{\prime}$ is a subfield of $S^{\prime}, R=\sigma^{-1}\left(F^{\prime}\right)$ is a weak primary subring of $S$ of exponent $\mu$. Further, there exists an isomorphism $\tau$ of the residue field of $R$ onto $F^{\prime}$, and, if $\phi$ denotes the natural mapping of $R$ onto its residue field, then $\tau \phi$ gives the restriction of $\sigma$ to $R$.

Proof. Clearly $R$ is a subring of $S$.

Let $x$ be any non-unit of $R$. Then $x$ must belong to the maximal ideal of $S$, since otherwise there would exist $y \in S$ such that $x y=1$, and hence such that $\sigma(x) \sigma(y)=1$. This would imply that $\sigma(y) \in F^{\prime}$, from which it would follow that $y \in R$. Accordingly we should have $x y=1$, with $y \in R$, contradicting the fact that $x$ is a non-unit of $R$. Consequently, if $\lambda$ is the exponent of $S$, then $x^{\lambda}=0$, so that $\{\sigma(x)\}^{\lambda}=0$; hence $\sigma(x)=0$, since $F^{\prime}$ is a field. This proves that $x$, any non-unit of $R$, belongs to the kernel, say $a$, of $\sigma$. Combining this with the fact that $a$ is necessarily a proper ideal of $R$, we see that a consists of all the non-units of $R$. Now

$$
\mathfrak{a}^{\mu}=(0), \quad a^{\nu} \neq(0) \text { if } \nu<\mu,
$$

from which we deduce that $R$ is a weak primary ring of exponent $\mu$.

To prove the last part of the lemma, we observe that $\sigma$ induces a homomorphism of $R$ onto $F^{\prime \prime}$ with kernel $a$. Thus there exists an isomorphism $\tau$ of $R /$ a (i.e. the residue field of $R$ ) onto $F^{\prime}$. By the definition of $\tau$, we have, for any $x \in R, \tau \phi(x)=\sigma(x)$; consequently $\tau \phi=\sigma$ on $R$.

LEMMA 4. Let ' $T$ ' be a commutative ring with an identity element, and let $S, S^{\prime}$ be weak primary rings which present the equicharacteristic case. Let $\theta, \theta^{\prime}$ be homomorphisms of $T$ onto $S, S^{\prime}$, respectively, and assume that there exists a homomorphism $\sigma$ of $S$ onto $S^{\prime}$ such that

(i) $\theta^{\prime}=\sigma \theta$, and

(ii) if $\mu$ is the exponent of the kernel of $\sigma$, then $\mu \leqslant 2$ when $S$ has characteristic zero and $\mu \leqslant p$ when it has characteristic $p(>0)$.

Let $K$ be a subfield of $T$, and $F^{\prime}$ a subfield of $S^{\prime}$ which contains $\theta^{\prime}(K)$. Then, if $F^{\prime \prime}$ is separable over $\theta^{\prime}(K)$, there exists an extension field $F$ of $\theta(K)$ in $S$ such that $\sigma(F)=F^{\prime}$. Further, $F$ is a separable extension of $\theta(K)$.

Proof. Let $R=\sigma^{-1}\left(F^{\prime}\right)$; then, by Lemma $3, R$ is a weak primary subring of $S$, whose exponent $\mu$ satisfies the conditions of Lemma 2 ; and

$$
\sigma \theta(K)=\theta^{\prime}(K) \subseteq F^{\prime}, \text { so that } \theta(K) \subseteq \sigma^{-1}\left(F^{\prime}\right)=R,
$$

showing that $\theta(K)$ is a subfield of $R$. Moreover (Lemma 3), there exists an isomorphism $\tau$ of the residue field, say $E$, of $R$ onto $F^{\prime}$; consequently, since $F^{\prime}$ is separable over $\theta^{\prime}(K)$, we find that $\tau^{-1}\left(F^{\prime}\right)(=E)$ is separable over $\tau^{-1} \theta^{\prime}(K)$, i.e. over $\tau^{-1} \sigma \theta(K)$. But, by Lemma $3, \tau^{-1} \sigma=\phi$ 
on $R$, where $\phi$ is the natural mapping of $R$ onto $E$; accordingly $E$ is separable over $\phi \theta(K)$. Hence, by Lemma $2, \theta(K)$ can be extended to a coefficient field, say $F$, of $R$, and we have

$$
\sigma(F)=\tau \phi(F)=\tau(E)=F^{\prime},
$$

as required.

Finally, $F$ is separable over $\theta(K)$ since $\sigma$ induces an isomorphism of $F$ onto $F^{\prime}$, and under this isomorphism $\theta(K)$ corresponds to $\theta^{\prime}(K)$.

3. The principal result. Let $Q$ be a weak local ring with maximal ideal $\mathrm{m}$. Denote by $Q_{i}$ the weak primary ring $Q / \mathrm{m}^{i}$, and let $\theta_{i}$ and $\sigma_{i}$ be the natural homomorphisms of $Q$ onto $Q_{i}$ and of $Q_{i+1}$ onto $Q_{i}$, respectively, for all $i=1,2,3, \ldots$. Then it is clear that $\theta_{i}=\sigma_{i} \theta_{i+1}$. Further, the kernel of the homomorphism $\sigma_{i}$ is $\mathrm{m}^{i} / \mathrm{m}^{i+1}$, and so has exponent 1 or 2. (The exponent may be 1 if $Q$ is a weak primary ring.)

We note that $Q_{1}$ is the residue field of $Q$, and that $\theta_{1}$ is the natural homomorphism of $Q$ onto its residue field.

In proving the theorem below, we shall essentially make use of the projective limit of the $Q_{i}$. We have already applied this notion to a situation similar to the above in [3] and [4], but here we shall use the concept in a slightly different form, in order to show precisely where the completeness of the ring is required.

We first prove

LEMMa 5. If $x$ is an element of a weak local ring (not necessarily complete) such that $\theta_{i}(x)=0$ for all $i$, then $x=0$.

Proof. We have $x \in \mathrm{m}^{i}$ for all $i$, so that

$$
x \in \bigcap_{i=1}^{\infty} m^{i}=(0)
$$

The following result is contained in Lemma 5 of [3].

LEMMA 6. If $Q$ is a complete weak local ring and if $\left\{x_{i}\right\}$ is a sequence such that $x_{i} \in Q_{i}$ and $\sigma_{i}\left(x_{i+1}\right)=x_{i}$ for all $i$, then there exists an element $x \in Q$ such that $\theta_{i}(x)=x_{i}$ for all $i$.

We now establish our main result.

THEOREM 1. Let $Q$ be a complete weak local ring with $K$ as a subfield. Suppose that $F_{1}$ is a separable field extension of $\theta_{1}(K)$ in $Q_{1}$. Then there exists an extension field $F$ of $K$ in $Q$ such that $\theta_{1}(F)=F_{1}$.

Proof. We first note that, from the observations made at the beginning of this section, the conditions of Lemma 4 are satisfied if we take

$$
T=Q, S=Q_{2}, S^{\prime}=Q_{1}, \theta=\theta_{2}, \theta^{\prime}=\theta_{1} .
$$

Accordingly we can find a subfield $F_{2}$ of $Q_{2}$ such that $\theta_{2}(K) \subseteq F_{2}$ and $\sigma_{1}\left(F_{2}\right)=F_{1} ;$ and $F_{2}$ is separable over $\theta_{2}(K)$, i.e. over $\sigma_{2} \theta_{3}(K)$. Hence, again by our earlier observations and Lemma 4 with

$$
T^{\prime}=Q, S=Q_{3}, S^{\prime}=Q_{2}, \theta=\theta_{3}, \theta^{\prime}=\theta_{2},
$$

there exists a subfield $F_{3}$ of $Q_{3}$ such that $\theta_{3}(K) \subseteq F_{3}, \sigma_{2}\left(F_{3}\right)=F_{2}$, and $F_{3}$ is separable over $\theta_{3}(K)$. We continue in this way to determine a sequence $\left\{F_{i}\right\}$ such that $F_{i}$ is a subfield of $Q_{i}$ and 


$$
\theta_{i}(K) \subseteq F_{i}, \sigma_{i}\left(F_{i+1}\right)=F_{i}
$$

Suppose now that $Q$ is a weak primary ring of exponent $\lambda$. Then, after $\lambda-1$ applications of the above procedure, we obtain $\theta_{\lambda}(K) \subseteq F_{\lambda}$, i.e. $K \subseteq F_{\lambda}$, and

$$
\begin{aligned}
\theta_{1}\left(F_{\lambda}\right) & =\sigma_{1} \sigma_{2} \ldots \sigma_{\lambda-1}\left(F_{\lambda}\right) \\
& =\sigma_{1} \sigma_{2} \ldots \sigma_{\lambda-2}\left(F_{\lambda-1}\right)=\ldots=F_{1} .
\end{aligned}
$$

If we now take $F=F_{\lambda}$, we obtain a subfield of the required type.

Consider now the general case. Let $F$ denote the set consisting of all elements $x \in Q$ such that $\theta_{i}(x) \in F_{i}$ for all $i$. It is clear that $F$ is a subring of $Q$. Let $x \in F$, and assume that $x \neq 0$. We assert that $\theta_{1}(x) \neq 0$. Suppose the contrary. Then, from $\theta_{1}=\sigma_{1} \theta_{2}$ and the fact that $\sigma_{1}$ induces an isomorphism of $F_{2}$ onto $F_{1}$, we obtain $\theta_{2}(x)=0$; and similarly

$$
\theta_{3}(x)=\theta_{4}(x)=\ldots=0 .
$$

Thus $\theta_{i}(x)=0$ for all $i$, so that, by Lemma $5, x=0$, contrary to hypothesis. From this it follows that $x \notin \mathrm{m}$; accordingly there exists $y \in Q$ such that $x y=1$, and hence, for any $i$, $\theta_{i}(x) \theta_{i}(y)=1$. But, since $\theta_{i}(x) \epsilon F_{i}$ and $F_{i}$ is a field, this implies that $\theta_{i}(y) \in F_{i}$ for all $i$, i.e. that $y \in F$. This proves that any non-zero element of $F$ possesses an inverse in $F$, and establishes that $F$ is a field.

To complete the proof, we now show that $\theta_{1}(F)=F_{1}$, and we first observe that, from the definition of $F$, it follows that $\theta_{1}(F) \subseteq F_{1}$. Assume now that $x_{1}$ is any element of $F_{1}$; then, since $\sigma_{i}\left(F_{i+1}\right)=F_{i}$ for all $i$, we can determine a sequence $\left\{x_{i}\right\}$ such that $x_{i} \in F_{i}$ and $\sigma_{i}\left(x_{i+1}\right)=x_{i}$. Accordingly, by Lemma 6 , $\dagger$ there exists $x \in Q$ such that $\theta_{i}(x)=x_{i}$ for all $i$. Since $x_{i} \in F_{i}$, we in fact have $x \in F$, by the definition of $F$, and this gives $x_{1}=\theta_{1}(x) \in \theta_{1}(F)$, showing that $F_{1} \subseteq \theta_{1}(F)$. Thus $\theta_{1}(F)=F_{1}$, as required.

COROLlary 1. Let $Q$ be a complete weak local ring, and let $K$ be a subfield of $Q$ such that the residue field of $Q$ is separable over $\theta_{1}(K)$. Then $K$ can be extended to a coefficient field of $Q . \ddagger$

Proof. In the theorem, take $F_{1}$ to be the residue field.

Corollary 2 (The Embedding Theorem). Any complete equicharacteristic weak local ring $Q$ possesses a coefficient field.

Proof. If $Q$ has characteristic zero, then $Q$ contains an isomorph $K$ of the rational field ; while, if $Q$ has prime characteristic, the integral multiples of the identity form a subfield $K$ of $Q$ which is perfect. Now any extension of a field of characteristic zero is a separable extension, and it may be shown (see, e.g., [5], p. 113) that any extension of a perfect field is a separable extension. Thus, in either case, $K$ can be extended to a coefficient field of $Q$ (Corollary 1 ), so that a fortiori $Q$ possesses a coefficient field.

Cororidary 3. Any maximal subfield of a complete (equicharacteristic) weak local ring of characteristic zero is a coefficient field. $\S$

Proof. Let $K$ be a maximal subfield ; then, since the characteristic is zero, $K$ can be extended to a coefficient field (Corollary 1), which must coincide with $K$ because of the maximality of $K$.

$\dagger$ It is at this stage that the completeness of $Q$ is required.

$\ddagger$ Chevalley [1] has proved this result for the case in which $Q$ is a complete local ring and the residue field is separable over $\theta_{1}(K)$ in the classical sense.

$\S$ This result has been established by Cohen [2] for complete local rings. 


\section{A. GEDDES}

\section{REFERENCES}

1. C. Chevalley, On the theory of local rings, Ann. Math., 44 (1943), 690-708.

2. I. S. Cohen, On the structure and ideal theory of complete local rings, Trans. Amer. Math. Soc., 59 (1946), 54-106.

3. A. Geddes, A short proof of the existence of coefficient fields for complete equicharacteristic local rings, J. London Math. Soc., 29 (1954), 334-341.

4. A. Geddes, On the embedding theorems for complete local rings, Proc. London Math. Soc. (3), 6 (1956), 343-354.

5. O. Zariski and P. Samuel, Commutative Algebra (Volume I) (Princeton, 1958).

The UnIversity

Glasgow 